\title{
A Comparative Analysis of the Predictive Abilities of Economic Complexity Metrics Using International Trade Network
}

\author{
Hao Liao $\mathbb{D}^{1}$ and Alexandre Vidmer $\mathbb{D}^{1,2}$ \\ ${ }^{1}$ National Engineering Laboratory for Big Data System Computing Technology, Guangdong Province Key Laboratory of Popular High \\ Performance Computers, College of Computer Science and Software Engineering, Shenzhen University, Shenzhen 518060, China \\ ${ }^{2}$ Department of Physics, University of Fribourg, 1700 Fribourg, Switzerland
}

Correspondence should be addressed to Hao Liao; jamesliao520@gmail.com and Alexandre Vidmer; alexandre@vidmer.com

Received 18 August 2017; Revised 8 December 2017; Accepted 27 December 2017; Published 13 February 2018

Academic Editor: Tiziano Squartini

Copyright (C) 2018 Hao Liao and Alexandre Vidmer. This is an open access article distributed under the Creative Commons Attribution License, which permits unrestricted use, distribution, and reproduction in any medium, provided the original work is properly cited.

\begin{abstract}
The complex networks approach has proven to be an effective tool to understand and predict the evolution of a wide range of complex systems. In this work, we consider the network representing the exchange of goods between countries: the international trade network. According to the type of goods they export, the complex networks approach allows inferring which countries will have a bigger growth compared to others. The aim of this work is to study three different methods characterizing the complex networks and study their behaviour on two main topics. Can the method predict the economic evolution of a country? What happens to those methods when we merge the economies?
\end{abstract}

\section{Introduction}

The development of countries is a crucial question in economics. In the next ten years, which countries will grow and develop the biggest industries? The GDP per capita is one of the main criteria to assess a country's development. Even though it does not account for the wealth distribution or for the purchasing power of the countries, it is a good indicator of the country's wealth and especially of its industrial sector. Twice a year, in April and in October, the International Monetary Fund (IMF) makes projection for the future GDP growth rate of countries. They do not detail the precise procedure; however they indicate that they use many factors to do the predictions. Actually, the prediction is done in a different way for each country and is then aggregated and readjusted in order to harmonize the projections. The predictions performed using complex networks do not outperform the projections made by the IMF, but their goal is to make additional information available that an organization such as the IMF could benefit from by taking into account their factors.

Two methods, the Method of Reflections [1] and Fitness and Complexity [2], were designed to rank the countries according to their potential growth. The two methods use a complex network approach to perform the ranking. The networks are built according to the revealed comparative advantage (RCA) procedure [3] described in the datasets section. The Method of Reflections proposed the Economic Complexity Index (ECI) to account for the production characteristics of countries, and the authors claimed it to be a better predictor than existing governance, institution, education quality, and economic competitiveness indexes [4]. However, there is also some criticism towards the Method of Reflections; $[2,5,6]$ point out that the Method of Reflections always underestimates the important counties with high diversification in exports, such as China and India. Moreover, the ECI definition based on the second eigenvector has been firstly shown in [7]. The Fitness and Complexity method was designed to take this feature into account and was featured in Nature news (http://www.nature.com/news/physicists-makeweather-forecasts-for-economies-1.16963).

In this paper, we study three metrics, Fitness and Complexity, the Method of Reflections, and the simple sum of different exports for each country. We review their potential to predict the economic growth of countries in detail and show 
that the Fitness and Complexity is the best method to rank the countries and their exports according to their importance. We push the study further to show one additional aspect of the Fitness and Complexity approach by merging countries' economies. We show that this approach captures additional features of the data that cannot be performed by a simpler approach.

\section{Materials and Methods}

2.1. International Trade Network. Two different datasets are used in this work. The first one ranges from 1998 to 2014 and has been cleaned by the BACI team [8]. The data were taken from [9]. The second dataset ranged from 1962 to 2000 and is used for the study of countries' merging and separation, as there are several occurrences during this period. This dataset was cleaned and processed by [10]. Both datasets are represented as bipartite in which one type of node represents the countries and the other type represents the products.

1962 to 2000. This dataset is very complete. The documentation lists all the operations that were made on the data, as well as the problems we might encounter. The gathering of the data would seem to be quite easy, as exports are likely to be registered somewhere. However this is not always the case, for three main reasons. First, even if we use the word product in order to simplify the discussion, only the category of products is specified in the data. Some examples of categories are "domestic, nonelectric, heating, cooking apparatus, and parts," "television receivers and color," and "office and stationary supplies, of base metal." This is a very rough categorization, which can contain products of various qualities. Some cooking apparatus will break after two uses while a fine quality one will last for decades. This is also a problem when the categories change. In 1984, the classification of products was revised, and so new categories appeared in the dataset. In order to avoid troubles linked to new categorization, we only use data from 1984 to 2000.

Secondly, a country (or someone working at the customs) can misreport a product assigning it to the wrong category or just misreport the quantity of export. The choice was made in [10] to give more credibility to the importer reports over the exporter reports, as they believe it would be more accurate. This makes sense if we consider that countries have a tax on imports and so have an interest in an accurate reporting. When the importer report is not available however, the exporter report is then considered. The misreports for categories were treated by creating additional categories called "aggregate categories." In our work, we exclude these aggregates.

The last reason is the absence of many countries' reports. In the paper, 72 countries reported to the UN database. However, we can still find information about the other countries by considering their trade with the 72 base countries. Some information is of course missing, as we know nothing about the trade between two countries that are not part of the 72 that reported. We can choose to include only 72 countries, or take all of them into account. The main trouble that arises if we exclude countries is that some low complexity objects (e.g., soy) are considered as high complexity ones because they are only seldom exported by the 72 countries, while being low complexity products (being easy to produce compared to a last-generation smartphone). Nonetheless, previous studies have used data from both countries inside and outside the core and obtained coherent results $[11,12]$.

The products can be categorized with different number of digits, but we keep only the categories at 4 digits in order to avoid too many unique items. We discard every product that is not in the official classification, as they were aggregately added by the authors to make the numbers more consistent. These products are easy to identify as they end with letters A and $\mathrm{X}$ or a 0 in the dataset.

1998 to 2014. This dataset was cleaned using harmonization techniques in [8]. Contrary to the previous dataset, the authors try to reconcile the exporter reports with the importer reports. When a trade is reported, it is usually done adding the transport cost on the exporter side, while the importer does not include this cost. The authors then evaluate the transport cost using a gravity equation depending on the distance between the two countries. We use this dataset to study the recent and the future evolution of countries, as the data reported in this dataset is more complete. Since the year 2000, approximately 150 countries have adopted the classification used in these data as legal instrument to report their trade.

$R C A$. In the international trade network, the information consists of countries' exports to other countries. We take a simplified approach here and only consider the total volume of export in US\$ of individual products that a country exports, and by extension we refer to it as its production.

We represent the data as a bipartite network for which one type of nodes is countries while the other one is products. Obviously, if a country exports only a tiny amount of a product, it should not be considered as an exporter of this product. The export of a product should be a reasonable fraction of the economy of the country, and the country should also export a reasonable fraction of the global export of the product. We define a clear boundary to choose whether a country is an exporter of a product or not by considering the "revealed comparative advantage" (RCA) [3]:

$$
\mathrm{RCA}_{i \alpha}=\frac{e_{i \alpha} / \sum_{j} e_{j \alpha}}{\sum_{\beta} e_{i \beta} / \sum_{j \beta} e_{i \beta}},
$$

where $e_{i \alpha}$ is the export volume of country $i$ for product $\alpha$ in US\$. We consider that country $i$ is an exporter of good $\alpha$ only if $\mathrm{RCA}_{i \alpha} \geq 1$. This ratio determines the relative importance of an export for a country and compares it with its relative importance for other countries.

Let us take an intuitive example to illustrate the sense of this metric, based on the values of year 1998 found in the dataset created by [10]. We compare the relative importance of chocolate exports for France and Switzerland in Table 1. Though France exports twice more chocolate than Switzerland, it is still considered to have less advantage in the export of chocolate. This is due to the fact that France exports much more other goods than Switzerland, so it is expected 
TABLE 1: Illustration using RCA of chocolate for France and Switzerland. The data are taken from 1998 and the values are given in billions of US\$ [10].

\begin{tabular}{lccc}
\hline & Total of all exports & Total for chocolate & RCA \\
\hline France & 292 & 0.68 & 1.71 \\
Switzerland & 83 & 0.29 & 2.60 \\
World & 5209 & 7.1 & - \\
\hline
\end{tabular}

that its exports are on average higher than those of Switzerland.

After applying the RCA threshold to the international trade, we can build the international trade network. Instead of the numerous pieces of information available before the procedure, we are left with binaries information: does this country export this product? Based solely on this simplified data, we show how we are able to extract relevant information in the next sections.

2.2. Methods. One of the first approaches to devise a score to rank countries and products in the international trade network was done in [1]. The algorithm is iterative and is given at step $n$ by

$$
\begin{aligned}
k_{i}^{(n)} & =\frac{1}{k_{i}} \sum_{\alpha \in \mathcal{N}(i)} k_{\alpha}^{(n-1)} \\
k_{\alpha}^{(n)} & =\frac{1}{k_{\alpha}} \sum_{i \in \mathcal{N}(\alpha)} k_{i}^{(n-1)},
\end{aligned}
$$

where $k_{i}^{(n)}$ is the score of country $i$ and $k_{\alpha}^{(n)}$ the score of country $\alpha$. Both scores are initialized with degree $\left(k_{i}^{(0)}=k_{i}\right.$ and $k_{\alpha}^{(0)}=k_{\alpha}$ ). In the original method, a threshold value is set, and when the total change on the scores is smaller than this value, the computation stops. The choice of the threshold is important, as the scores all converge to a trivial fixed point. The threshold has to be set big enough so that the differences between scores do not exceed machine precision; this point is discussed in [6]. Note that a definition based on eigenvectors is given in [4] and provides similar results but gets rid of the need to define a threshold. However, it has been noted in [13] that stopping the computation after two iterations gives the most coherent results according to our current knowledge of the network, as the correlation between the diversification of a country and its score reduces after additional iterations [6]. We then make the choice of stopping the algorithm after only two iterations. We consider here only the deviations to the average values obtained with the algorithm and label the final scores $\mathrm{MR}_{i}^{c}$ and $\mathrm{MR}_{\alpha}^{p}$ for country $i$ and product $\alpha$, respectively:

$$
\begin{aligned}
\mathrm{MR}_{i}^{c} & =\frac{\widetilde{\mathrm{MR}}_{i}^{c}-\left\langle\widetilde{\mathrm{MR}}^{c}\right\rangle}{\operatorname{std}\left(\widetilde{\mathrm{MR}}^{c}\right)} \\
\mathrm{MR}_{\alpha}^{p} & =\frac{\widetilde{\mathrm{MR}}_{\alpha}^{p}-\left\langle\widetilde{\mathrm{MR}}^{p}\right\rangle}{\operatorname{std}\left(\widetilde{\mathrm{MR}}^{p}\right)} .
\end{aligned}
$$

$\widetilde{\mathrm{MR}}_{i}^{c}$ is the value of $k_{i}^{(2)}$ and similarly $\widetilde{\mathrm{MR}}^{p}$ corresponds to $k_{\alpha}^{(2)}$. $\left\langle\widetilde{\mathrm{MR}}^{c}\right\rangle$ stands for average over all scores for countries, and $\operatorname{std}\left(\widehat{\mathrm{MR}}^{c}\right)$ its standard deviation. The score attributed to countries was shown to have a good predictive power with the economic growth compared to more traditional metrics [4].

The Fitness and Complexity metrics have been developed to measure the well-being of countries and their production [2]. The procedure has been shown to perform well in an ecological network, by ranking the species according to their importance in the sustainability of the system [14]. The algorithm has been generalized by exponentiating the product term [15]. The iterative self-consistent set of equations is defined as

$$
\begin{aligned}
F_{i}^{n} & =\sum_{\alpha \in \mathscr{C}_{i}} Q_{\alpha}^{n-1} \\
Q_{\alpha}^{n} & =\frac{1}{\sum_{i \in \mathscr{C}_{\alpha}} 1 / F_{i}^{n-1}},
\end{aligned}
$$

where $F_{i}^{n}$ is the fitness of country $i$ and $Q_{\alpha}^{n}$ the complexity of product $\alpha$ after $n$ iterations. The convergence of the algorithm and its stopping condition were studied in [15].

The idea behind the algorithm is that successful countries export complex products and complex products are only exported by successful countries. Indeed, the fitness of a country in (4) is simply the sum of the complexities of its exported goods. So a successful country should export many products, and these products should be of high complexity in order to achieve a high fitness. The complexity of a product in (4) is defined in a nonlinear way, which makes effectively the algorithm nonlinear. Due to the $1 / F_{i}^{n-1}$ factor in the equation, the complexity of a product is mainly characterized by the fitness of its lowest exporter. For instance, if we take a product $\alpha$ with two exporters $i$ and $j$ with fitness values of 0.1 and 10 , respectively, the complexity of the product would be 0.099 . If only country $i$ exports the product, the complexity would be 0.1 . The two values are close and we clearly see the dependence on the worst exporter. This makes sense for the interpretation of the score as if poor scoring country can export a good; this good should be of low complexity. And if only high scoring countries export a good, it should be hard to produce; otherwise some low fitness country would export it.

\section{Results}

In addition to the two metrics described previously, we add the degree for comparison (i.e., number of exports having a RCA equal to or above 1). The comparison with degree is lacking in the literature about Fitness and Complexity, and so we are interested in studying the additional information brought by the two iterative approaches. Indeed, previous works have shown a very high correlation between the degree of countries' exports and the fitness of these countries [13].

3.1. GDP Prediction. As mentioned in Introduction, the GDP growth is one of the main pieces of information about the economic performance of a country. In order to study the link between the metrics and the GDP evolution, we show in 
Figure 1 the trajectories of the countries in the GDP per capita plane coupled with the three different metrics [2] (degree, Methods of Reflections, and Fitness and Complexity). The data range from 1998 to 2014 [8]. We remark that the countries that lie in the region with high degree but somehow lower GDP per capita (such as China and India) tend to have a trajectory with growing GDP. The remark is also valid for fitness, but for Methods of Reflections it is less visible. The ones in the region with high GDP per capita compared to their degree (such as Norway and Iceland) tend to have a more static trajectory along the GDP per capita axis. This remark was made in [16] for fitness metric. It is hard to distinguish the different features between degree and Fitness on this graph; the same three countries (Czech Republic, China, and India) stand out. The exception is Philippines, which only stands out when looking at the Fitness metric. Its trajectory is quite flat for the moment as it just arrived in the supposed GDP growth region. It is hard to distinguish anything in the middle region of the graph, and so a coarse grained vision is needed in order to be able to see the tendencies.

Similarly to [16], we average the trajectories from 1998 to 2014 in Figure 3 for the three different metrics coupled with GDP per capita. In the degree plot, we see that every country which has a degree superior to about 100 has a significant GDP growth. The longest arrows can be observed in the far right region under the regression line in red, which means that the countries in this region experience the biggest growth. This is even more true in the panel with Fitness in Figure 3(c). The countries in the lower right part of the plot exhibit a growth significantly higher than in the other regions of the plane. In Methods of Reflections however, every arrow seems to point up at the same rate and it is hard to distinguish particular regions. By eye, the Fitness and Complexity metric seems to have more discriminating power than the degree, definitely more than Methods of Reflections. However, it is hard to draw any hard conclusion on the superiority of Fitness compared to degree based on these panels. But we will see in the next section in which domain the Fitness and Complexity metrics outperform the simple degree metric.

3.2. The Selective Predictability Scheme. In [16], a method based on the method of analogues $[17,18]$ was applied to the prediction of the countries' GDP. This method is named the selective predictability scheme. This method is useful in order to have a clear visualization of the tendencies. The average displacement in the plane presented in Figure 2 gives only a general tendency. But we can also ask the question: can we predict where the country will land in the plane in the future? If we can say with high probability where a country will be in the future according to its current position in the plane, it is also an interesting observation, while this may disappear in the averaging procedure used in the arrows picture. The evaluation goes as follows:

(i) The GDP-Fitness space in logarithmic plane is divided into boxes.

(ii) We report the box in which countries are at a given year and the one at which they end ten years later. (iii) The total number of countries inside box $i$ at year $t$ is $N_{t}^{i}$, and the number of different boxes occupied after ten years' evolution coming from box $i$ is labeled $n_{t}^{i}$.

(iv) For each box, we evaluate the quantity $\mathscr{C}_{t}^{i}=\left(n_{t}^{i} / N_{t}^{i}-\right.$ $\left.1 / N_{t}^{i}\right) /\left(1-1 / N_{t}^{i}\right)$. This quantity is related to the dispersion of the box. Indeed, if the evolution of countries is highly unpredictable, $n_{t}^{i}$ is large, and so $\mathscr{C}_{t}^{i}$ is large. Conversely, if countries tend to have a predictable evolution, they would end in the same boxes, and so $n_{t}^{i}$ would be small, resulting in a smaller value for $\mathscr{C}_{t}^{i}$.

The results for the three metrics are displayed in Figure 3. At first glance, it seems that degree and Fitness perform similarly, while Method of Reflections does not show exhibition and predictability. There are more boxes indicating low predictability (red) in the case of degree than for fitness. However there are also more boxes with high predictability (dark green). There are two shortcomings with the method of analogues. The first one is that the results are only graphical; it is then hard to say that a method is more adequate than another. The second one is that the results are dependent on the number of boxes we choose, as well as the range of the axis.

The method of analogues assigns a predictability score for the countries located inside specific boxes. Then, by summing over the predictability scores, the result should account for an overall predictability of the method. However, we need to get rid of the strong dependency on the choice of the number of boxes and their location. With this in mind, we propose to sum over all the boxes, taking into account the number of occupied boxes $N_{\text {occ }}$ as a parameter. The score is as follows:

$$
\mathscr{P}^{S}=\mathscr{P} \cdot \frac{2 \log \left(N_{\text {occ }}\right)}{n},
$$

where $\mathscr{P}=\sum_{i, t} 1-\mathscr{C}_{t}^{i}$ and $n$ is the number of events used during the computation, where an event is defined as the presence of a country in a box. The fact that $n$ is important is because we only take into account the events that are located in a box with at least five events. We choose to take the inverse of the dispersion in the sum, so that a high value of overall predictability $\mathscr{P}$ means that the prediction is accurate. The results are shown in Figure 4 as a function of the number of boxes occupied $N_{\text {occ }}$. The shadowed areas represent the standard deviation over 256 different choices of axis limits. The choice of the axis limits matters as the boxes are shifted depending on which values are comprised in the boxes. For instance, in case (a) the first interval is $[0.1,0.2]$ and in case (b) $[0.12,0.22]$. A dot located at 0.21 would be inside the first interval in case (b) but not in case (a). It is quite obvious that Methods of Reflections performs bad, especially that the results are highly dependent on the choice of axis' limits. While it is clear that the Method of Reflections has a poor predictability, it is clear that degree is performing better than Fitness and Complexity. We argue that this shows the predictive power of Fitness and Complexity is different from the one of diversification. Indeed, the score was developed to take into account the evolution of outliers. While diversification would grasp a better general trend, Fitness and Complexity method 

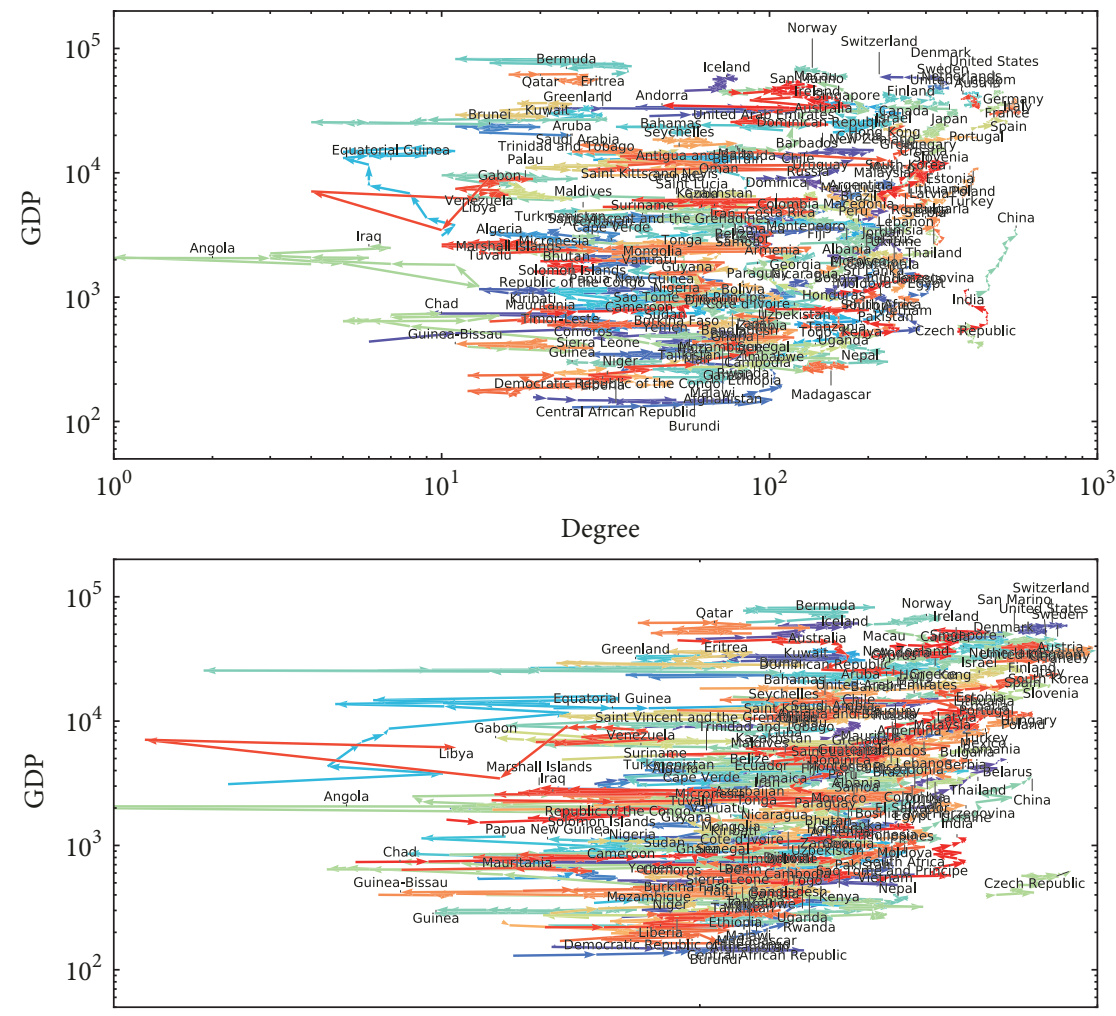

Method of reflections

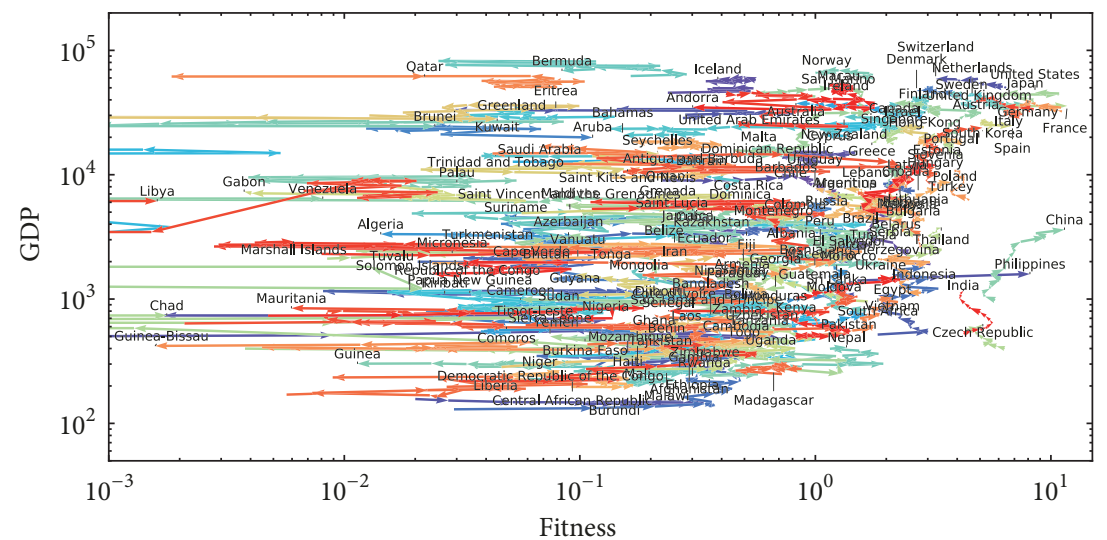

FIGURE 1: Evolution of the countries in the GDP per capita-score plane, where score can be degree, country's score obtained by Methods of Reflections or Fitness. The data we consider range from 1998 to 2014 and countries are assigned random colors for easier spotting of their respective trajectory.

has already proven to be a good predictor for countries in specific location [19].

\subsection{Effects of Countries' Unification on the Fitness of Countries.} What happens if countries decide to merge their exports? Will their fitness increase or will it decrease? This could go both ways, as the countries would lose some exports due to the RCA threshold: if the total export increases and the export for a specific product remains nearly constant, the RCA value can drop under one. Or in a different case if two countries export a lot to each other, they will lose a lot of exports when unifying. We show that some countries would benefit from a unification while some would worsen their fitness score. Fortunately, the unification and separation of countries happen several times in the dataset. We take the example of Germany, Czechoslovakia, and Yugoslavia to illustrate how the unification or separation of economies affects the fitness of countries. In a second time, we take artificial example to show that it is hard to predict which country would benefit from a unification and which one would not. This depends mainly on the details of their exports and not solely on their current fitness value.

In the original exports data, which country exports which products to another country is specified. While we chose to 


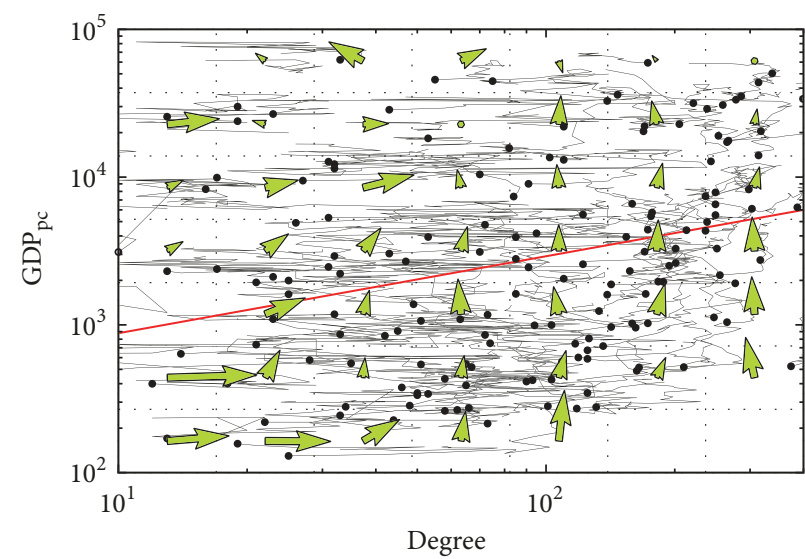

(a)

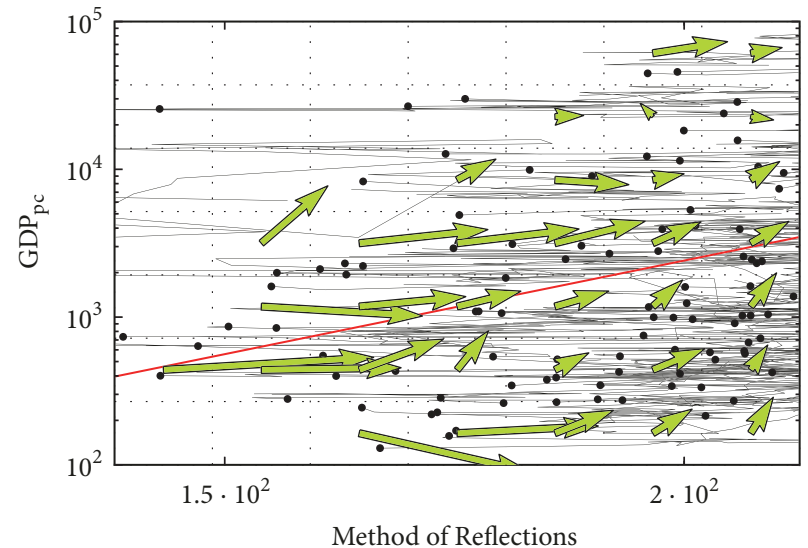

(b)

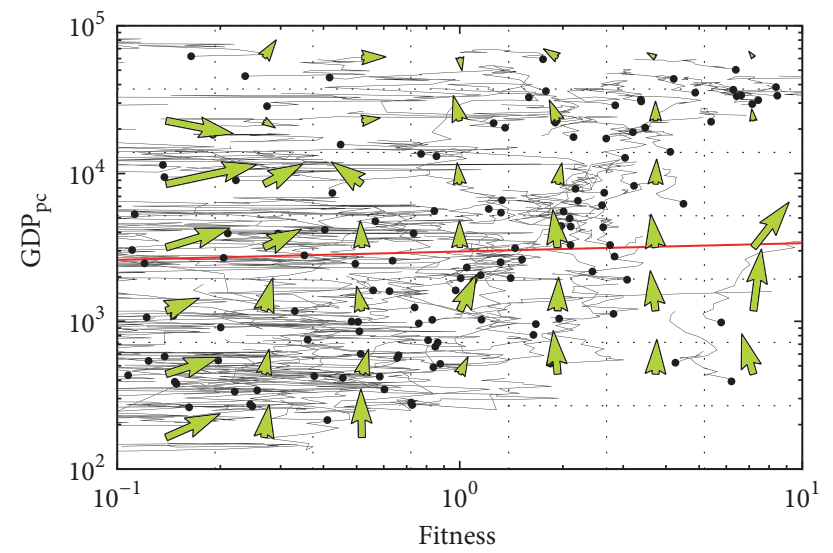

(c)

Figure 2: Average displacement in the GDP per capita and country score plane. The score of countries is degree in panel (a), Methods of Reflections in panel (b), and Fitness in panel (c). For a better view of the GDP change, the displacement along the GDP-axis is multiplied by 5 for a better visualization. The linear regression is shown by a red line for each metric.

simplify the data and take into account only the total exports for each product in the previous sections, the detailed data now prove useful when unifying the countries, as we are able to subtract the exports between them. We would like to stress before starting the analysis that we can only merge the exports of countries and not their ability to produce goods. Indeed, when two economies unify together, it is possible that their common capabilities on manufacturing can be combined to produce new goods or to increase significantly their export for a product $[1,6]$.

3.3.1. Real-World Examples. It happened several times during the world history that some countries decided to unify or that a country choose to separate into smaller ones. We investigate the case of separation and unification from a purely economic point of view, without considering any other factors. The question of interest here is to know whether the fitness of the countries increases or decreases in the process. If we theoretically unify two countries and the fitness of these two countries increases, the countries would have an economic benefit to unify. On the other hand, if the fitness of the countries decreases when theoretically unified, the country with the highest fitness would not benefit from the unification.

We study three real-world cases. The first is the reunification of East and West Germany in 1990. Nowadays, Germany is one of the top economies in the world; while we cannot study the imaginary economy that would result if current Germany would split again, we can merge the old East and West Germany. It is well-known that the West was more economically successful than the East in 1990. However, if the two economies are complementary, it is possible that the West could benefit from a merging as well. This is highly probable as the two parts of the countries were totally disconnected and the political systems were completely different. We show in Figure 5(a) the theoretical merge of the two Germanies. Not surprisingly, the fitness of West Germany is higher than the one of East Germany before 1990. We can see that the fitness of Germany starts to rise before 1990; this is due to the fact that data and some exports were referenced before the official classification; however the total export volume is really low. The curve named merge is the theoretical merge of the two Germanies. We observe that it is constantly higher than both the fitness of the West and East Germany. We could 


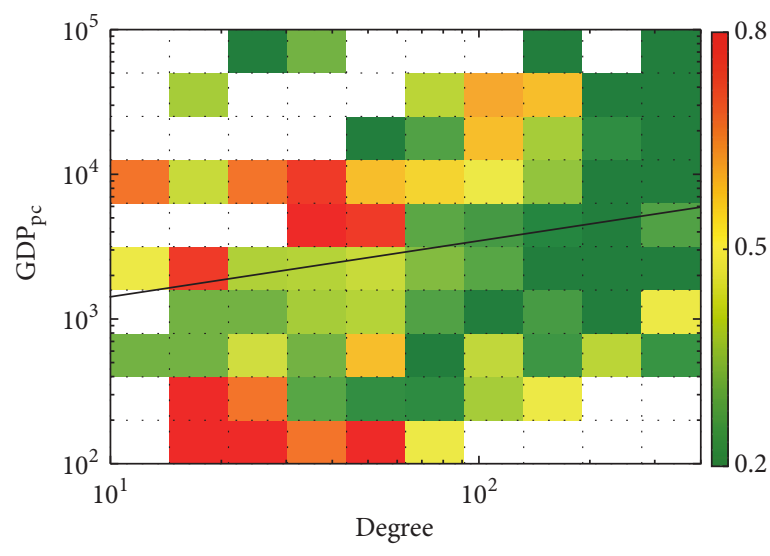

(a)

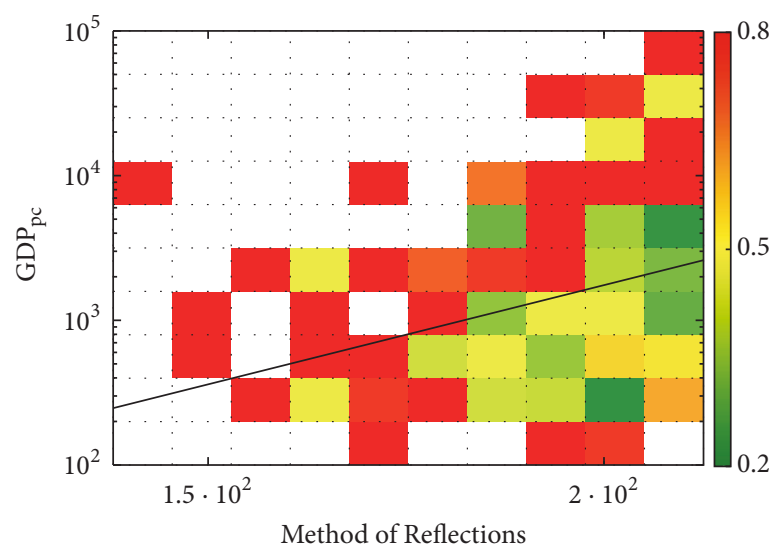

(b)

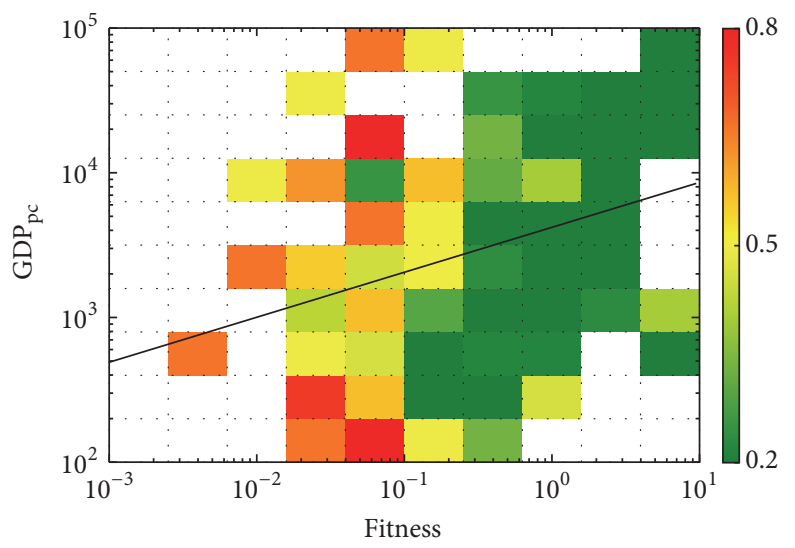

(c)

Figure 3: The selective predictability scheme with $N=10$ for the three different metrics. A low value means a low dispersion and so a high predictability, while a high value means unpredictability.

say that if the countries were to merge, they would benefit from it. And eventually they merged and became one of the strongest economies in the world, even the first according to the Fitness and Complexity metric. We do not show any confidence interval; however the robustness to noise is high and, most importantly, the tendency is consistent with years.

Our second example is the dissolution of Czechoslovakia into Czech Republic and Slovakia in 1992. The cause of the dissolution was mainly political, but there were also economic tensions [20]. The Slovaks had the impression that they were paying for the Czech, which seems to be true as the leading parties were mostly Czech and promoted Czech interests first. These tensions and the strong nationalism of Slovaks led to the separation of Czechoslovakia. The fitness of Czech Republic and Slovakia, Czechoslovakia before the dissolution, and the theoretical merge after the dissolution of Czechoslovakia are shown in Figure 5(b). In contrary to the Germany case, we see that the Czech Republic scores higher in the fitness metric than the merge of the two states. This is an interesting observation as we could claim that Czech Republic had a strong economic benefit in this dissolution.

The last example is the dissolution of Yugoslavia into Slovenia, Macedonia, Bosnia and Herzegovina, Albania, Federal Republic of Yugoslavia (Serbia and Montenegro), and
Croatia. According to [21], The Yugoslavian state, which was created after the first world war, was held together by having common enemies, especially Italy at that time. During the 20th century, there were many tensions in Europe and so the state stayed united in case of an attack. Due to the lack of pressure from the East, the ethnic tensions became stronger than the necessity of staying together against a stronger enemy. From 1991 to 1992, the state gradually separated into a multitude of smaller states. As for the two previous examples, the results are shown in Figure 5(c). Among all the states, only Slovenia benefited from the dissolution in terms of fitness. In a very short term, the Federal Republic of Yugoslavia also benefited from it, but the wars that created the dissolution certainly damaged strongly its economy.

In our three real-world examples, when two countries would benefit from merging, they end up by merging together. And when one country would benefit from a dissolution, they end up separating. We note that these are only anecdotal evidences, and there are factors affecting the dissolution or merging other than the economic factor. However the economy of a country in general reflects well its stability, its dynamism, and its well-being. As observed in the previous section about GDP, fitness does not necessarily add information when compared to degree. It is then natural to 

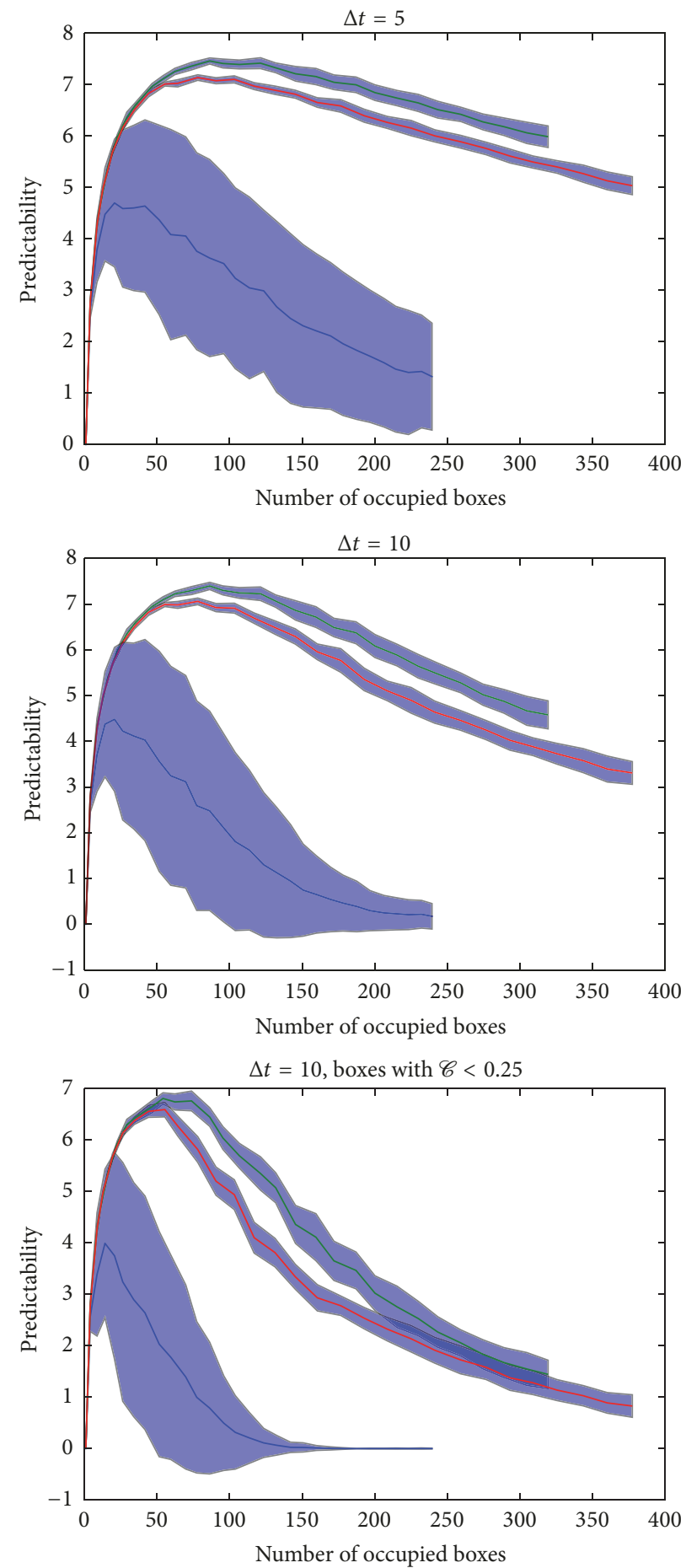

\section{- Method of Reflections \\ — Degree \\ _ Fitness}

FIGURE 4: Total predictability as a function of the number of boxes occupied $N_{\text {occ }}$ along one direction. The standard deviation is shown as shadowed area. The parameter $\Delta t$ stands for the evolution step; the countries start in a box and we observe in which one they end $\Delta t$ years later. A high score means a high predictability. 


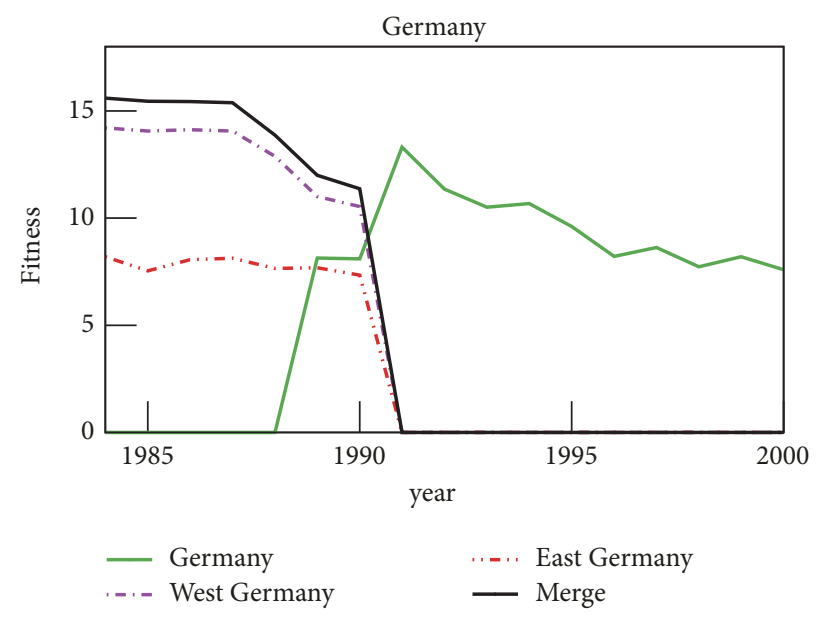

(a)

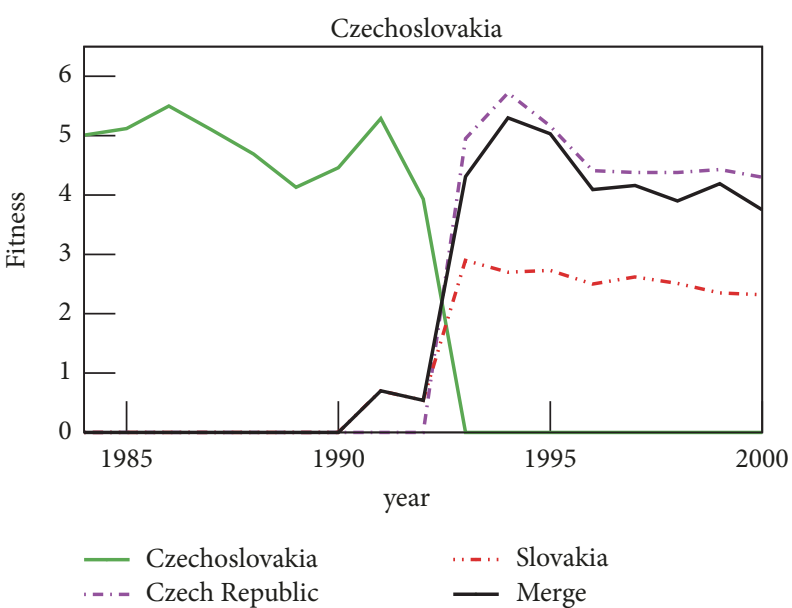

(b)

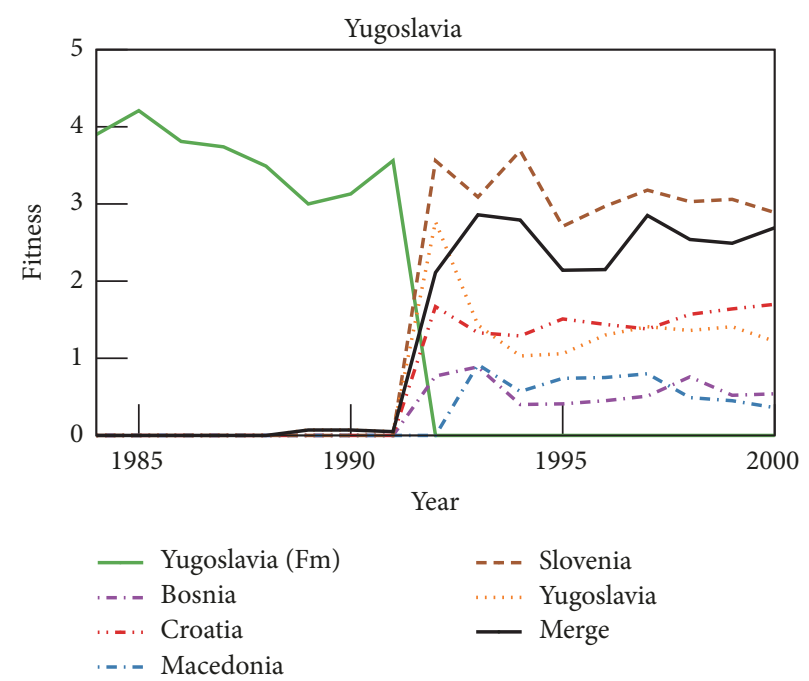

(c)

Figure 5: Comparison of countries' fitness values before they merge together or before they dissolve. The cases of Germany, Czechoslovakia, and Yugoslavia are presented in the panels.

ask the question: could we have obtained the same results by using only degree? We show the equivalent with degree in Figure 6. We see that it is in general less pronounced than with fitness and sometimes quite different. For instance, Slovenia performs worse than the merge when considering the degree metric, and West Germany performs very similarly to the merged Germanies. Another important point to note is the fact that the degree is nearly perfectly continuous and does not undergo tremendous variations. Conversely, Fitness shows some quick variations, which shows that it captures additional information that is hidden in a traditional approach.

3.3.2. Theoretical Cases. We saw in the previous section that if we merge economies together, the resulting economy can be either stronger or weaker in terms of fitness. But so far we did not investigate the mechanisms behind this result. In order to find the explanation for this phenomenon, we take fictitious example by merging one country with every other country in the dataset. By studying the relations between the countries when the fitness goes up, or goes down, we should be able to identify the common property between positive and negative merges.

We present the results of merging for four different countries in Figure 7. Obviously, it is not trivial to see if the fitness score of a country will rise or fall when merging. We see a clear dependency on the fitness of the target country; however it is only a tendency. For instance, Italy would benefit from merging with the two countries with the highest fitness scores (Germany and USA), while Japan would see its fitness decreasing. At the same time, the base countries rarely benefit from merging with a country with lower fitness. This is also interesting as it shows that this is quite rare and our previous example with West and East Germany is a special case. It is also surprising to see in the case of Italy that, even when merging with a country having a higher fitness, the fitness 


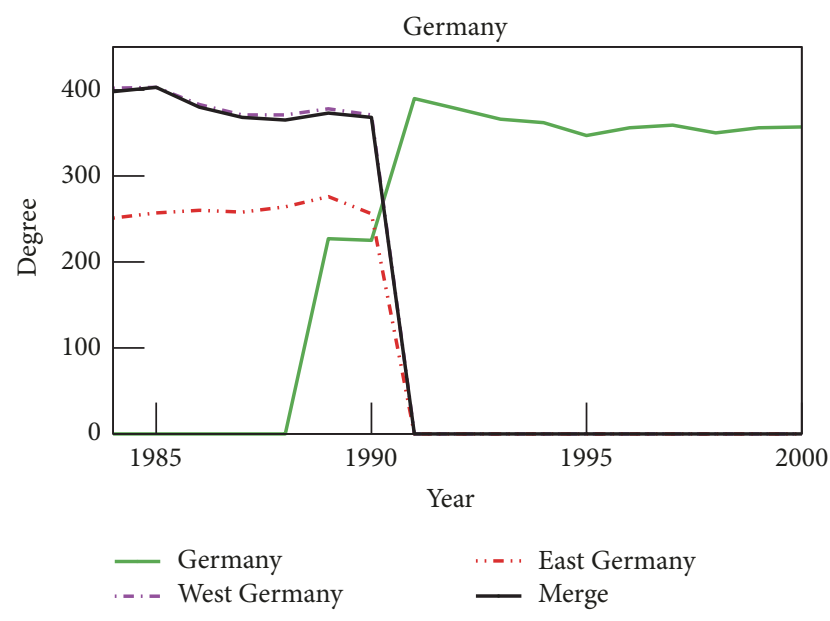

(a)

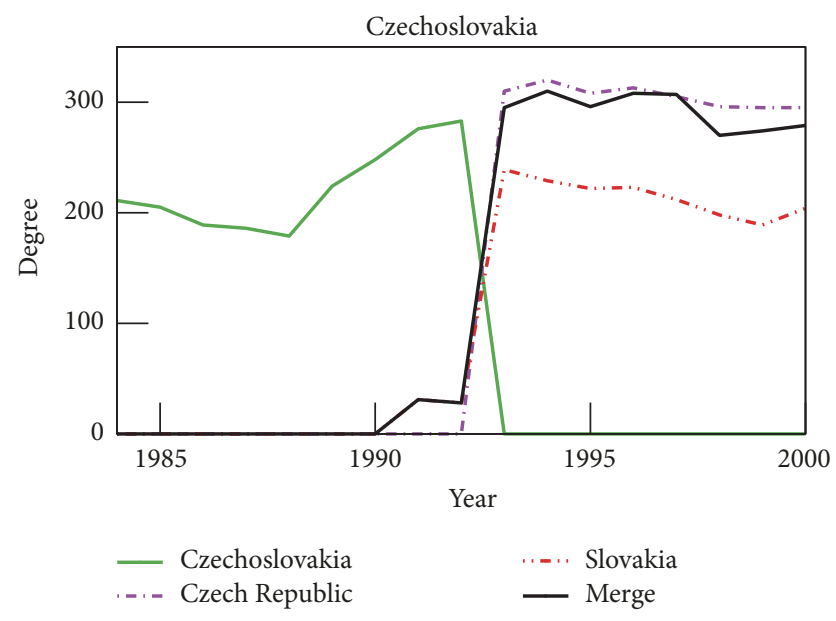

(b)

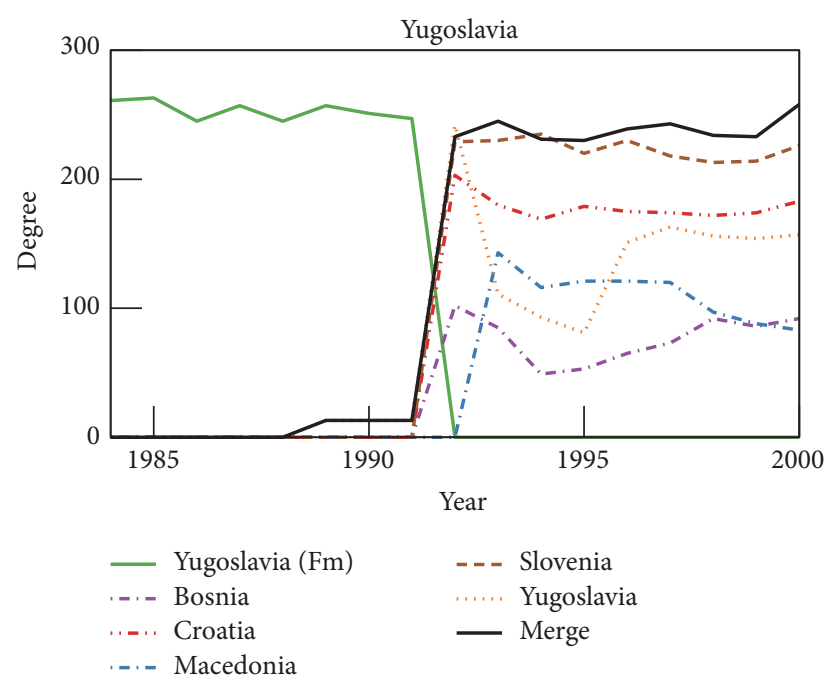

(c)

Figure 6: Comparison of countries' degrees before they merge together or before they dissolve. The cases of Germany, Czechoslovakia, and Yugoslavia are presented in the panels.

of Italy decreases significantly. This country that would make the fitness of Italy lower is actually France.

There is of course a dependency on the fitness of the target country, but it is not the only factor. As we see in Figure 7, the share of common exports is not an explanatory factor. However, we see that Japan would in general gain more fitness when merging with another country and that it has less common exports with other countries than the other three base countries. While we found no solid predictor to find if a country would benefit from a merge or not, it would definitely be linked with the rarity of their exports' basket. One possible way to investigate this problem would be to also take into account the complexity scores of the products they have or not in common. If they mostly share low complexity exports and have few high complexity products in common, a merge would add more high complexity products, while if they already share most high complexity products, a merge would not add many new products in the process.

\section{Conclusion}

Fitness and Complexity was shown to be the top performing algorithm to rank the countries and products according to their importance and allowed the exhibition of additional features compared to other approaches. While it is hard to conclude on its ability to predict economic growth of countries, it is undeniable that it captures additional information. In this work, we showed a way of comparing methods and a potential use of the methods' additional information. As shown in a previous study [22], the Fitness analysis can help the weak economies to understand how to exit the poverty trap, by increasing complexity of their exported products and increasing their diversification. Surprisingly, we saw that it is a good predictor for the economic results in case of the merging and separation of countries. While the analysis of the results remains a difficult task, a deeper understanding of the fitness change when two countries merge together or when a country 

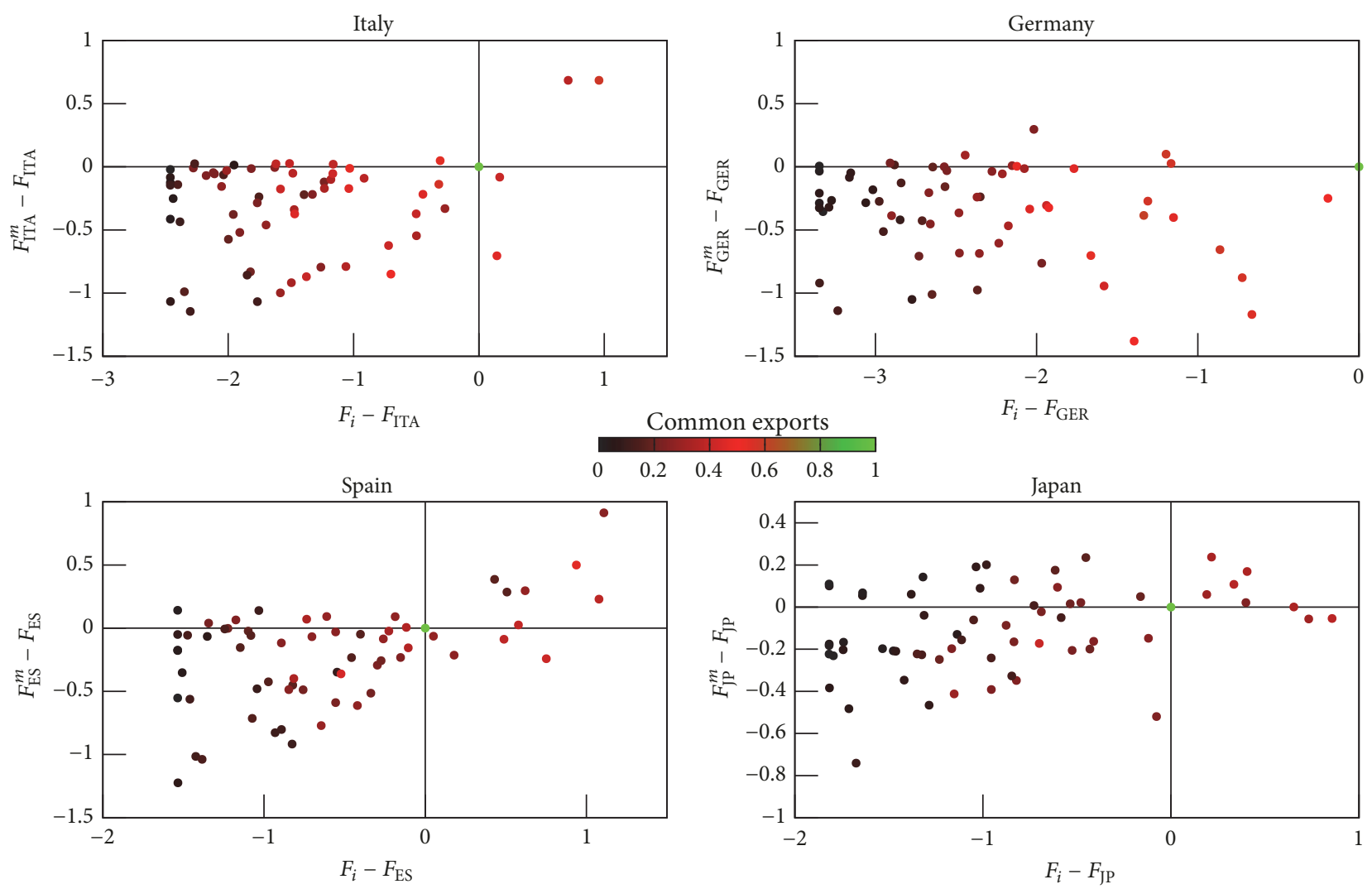

Figure 7: Merging of Italy, Germany, Spain, and Japan with all other countries in the dataset. The $x$-axis is the difference of fitness with the initial country and the target country it is merged with, while the $y$-axis is the difference of fitness when the initial country is merged with the target country. The color stands for the fraction of common exports between the base and target countries.

separates itself into smaller countries would tell us a lot about the metrics and the economic behaviour of countries.

\section{Conflicts of Interest}

There are no conflicts of interest.

\section{Acknowledgments}

The authors acknowledge financial support from the National Natural Science Foundation of China (Grant nos. 11547040 and 61703281), Guangdong Province Natural Science Foundation (Grant nos. 2016A030310051, 2017A030310374, and 2017B030314073), Shenzhen Fundamental Research Foundation (Grant nos. JCYJ20150625101524056, JCYJ20160520162743717, JCYJ20150529164656096, and JCYJ20170302153955969), Natural Science Foundation of SZU (Grant no. 2016-24), SKL-MCCS Open Fund (Project no. 20160104), Foundation for Distinguished Young Talents in Higher Education of Guangdong, China (Grant no. 2015KQNCX143), the EU FET-Open (Grant no. 611272 (project Growthcom)), and the Swiss National Science Foundation (Grant no. 200020-143272).

\section{References}

[1] C. A. Hidalgo and R. Hausmann, "The building blocks of economic complexity," Proceedings of the National Acadamy of Sciences of the United States of America, vol. 106, no. 26, pp. 10570-10575, 2009.

[2] A. Tacchella, M. Cristelli, G. Caldarelli, A. Gabrielli, and L. Pietronero, "A new metrics for countries' fitness and products' complexity," Scientific Reports, vol. 2, article 723, 2012.

[3] B. Balassa, "Trade Liberalisation and "Revealed" Comparative Advantage," The Manchester School, vol. 33, no. 2, pp. 99-123, 1965.

[4] R. Hausmann, C. A. Hidalgo, S. Bustos, M. Coscia, A. Simoes, and M. A. Yildirim, The Alas of Economic Complexity: Mapping Paths to Prosperity, MIT Press, 2014.

[5] L. Pietronero, M. Cristelli, A. Gabrielli et al., "Economic Complexity: "Buttarla in caciara" vs a constructive approach," Economics.

[6] M. Cristelli, A. Gabrielli, A. Tacchella, G. Caldarelli, and L. Pietronero, "Measuring the intangibles: a metrics for the economic complexity of countries and products," PLOS ONE, vol. 8, no. 8, Article ID e70726, 2013.

[7] G. Caldarelli, M. Cristelli, A. Gabrielli, L. Pietronero, A. Scala, and A. Tacchella, "A network analysis of countries' export flows: firm grounds for the building blocks of the economy," PLoS ONE, vol. 7, no. 10, Article ID e47278, 2012. 
[8] G. Gaulier and S. Zignago, "Baci: International trade database at the product-level. the 1994-2007 version," in Proceedings of the Working Papers 2010-23, CEPII, 2010, http://www.cepii.fr/ CEPII/fr/publications/wp/abstract.asp? NoDoc $=2726$.

[9] A. J. G. Simoes and C. A. Hidalgo, "The economic complexity observatory: An analytical tool for understanding the dynamics of economic development," in Scalable Integration of Analytics and Visualization, 2011.

[10] R. Feenstra, R. Lipsey, H. Deng, A. Ma, and H. Mo, "World Trade Flows: 1962-2000," National Bureau of Economic Research, 2005.

[11] C. A. Hidalgo, B. Winger, A.-L. Barabási, and R. Hausmann, "The product space conditions the development of nations," Science, vol. 317, no. 5837, pp. 482-487, 2007.

[12] R. Hausmann, J. Hwang, and D. Rodrik, "What you export matters," Journal of Economic Growth, vol. 12, no. 1, pp. 1-25, 2007.

[13] M. S. Mariani, A. Vidmer, M. Medo, and Y.-C. Zhang, "Measuring economic complexity of countries and products: which metric to use?" The European Physical Journal B, vol. 88, no. 11, article 293, pp. 1-9, 2015.

[14] V. Domínguez-García and M. A. Muñoz, "Ranking species in mutualistic networks," Scientific Reports, vol. 5, article 8182, 2015.

[15] E. Pugliese, A. Zaccaria, and L. Pietronero, "On the convergence of the Fitness-Complexity algorithm," The European Physical Journal Special Topics, vol. 225, no. 10, pp. 1893-1911, 2016.

[16] M. Cristelli, A. Tacchella, and L. Pietronero, "The heterogeneous dynamics of economic complexity," PLoS ONE, vol. 10, no. 2, Article ID e0117174, 2015.

[17] E. N. Lorenz, "Atmospheric predictability as revealed by naturally occurring analogs," Journal of the Atmospheric Sciences, vol. 26, no. 4, pp. 636-646, 1969.

[18] E. N. Lorenz, "Three approaches to atmospheric predictability," Bulletin of the American Meteorological Society, vol. 50, no. 3454, p. 349, 1969.

[19] M. Cristelli, A. Tacchella, L. Pietronero, and C. W. Chen, "The heterogeneous dynamics of economic complexity," PLoS ONE, vol. 10, no. 2, Article ID e0117174, pp. 1-15, 2015.

[20] P. S. Hilde, "Slovak nationalism and the break-up of Czechoslovakia," Europe - Asia Studies, vol. 51, no. 4, pp. 647-665, 1999.

[21] D. Sekulic, "The creation and dissolution of the multinational state: The case of Yugoslavia," Nations and Nationalism, vol. 3, no. 2, pp. 165-179, 1997.

[22] E. Pugliese, G. L. Chiarotti, A. Zaccaria, and L. Pietronero, "Complex economies have a lateral escape from the poverty trap," PLoS ONE, vol. 12, no. 1, Article ID e0168540, 2017. 


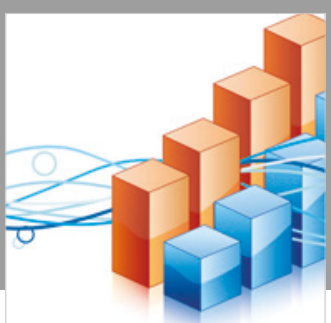

Advances in

Operations Research

\section{-n-m}
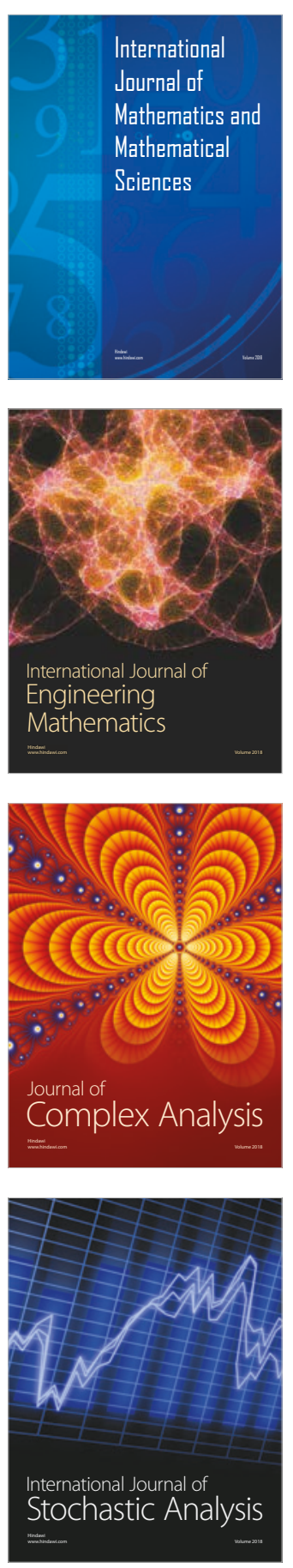
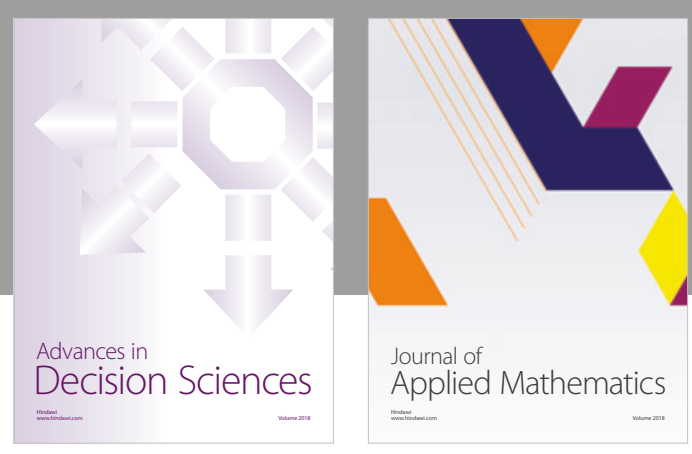

Journal of

Applied Mathematics
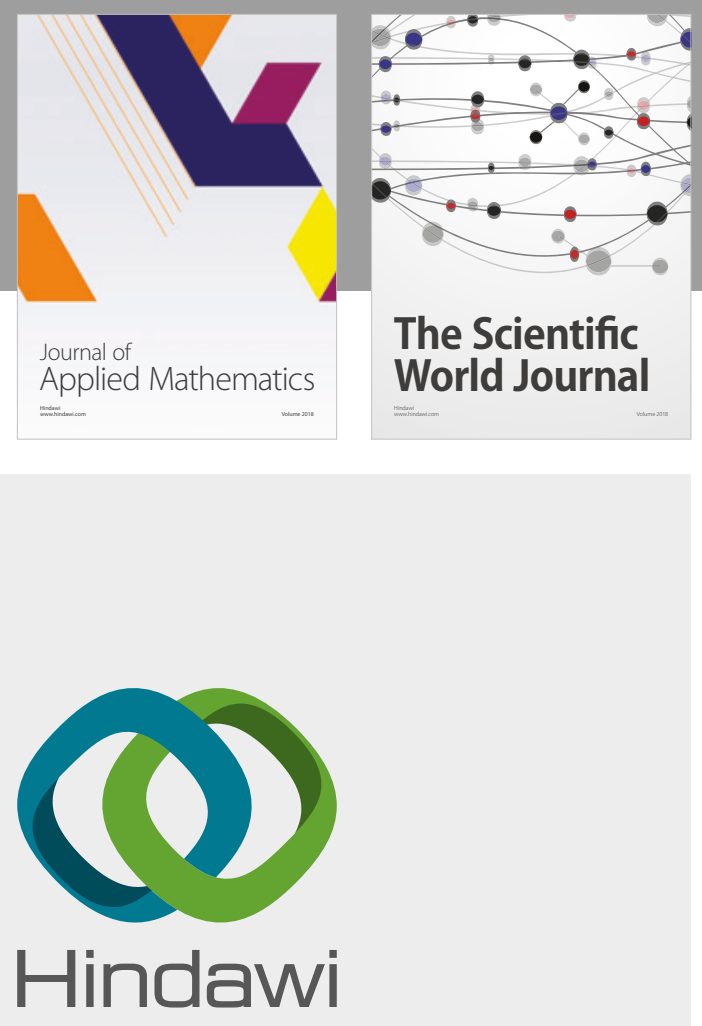

Submit your manuscripts at

www.hindawi.com

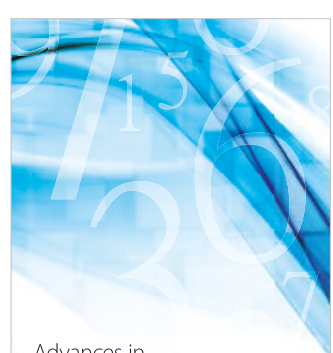

Advances in
Numerical Analysis
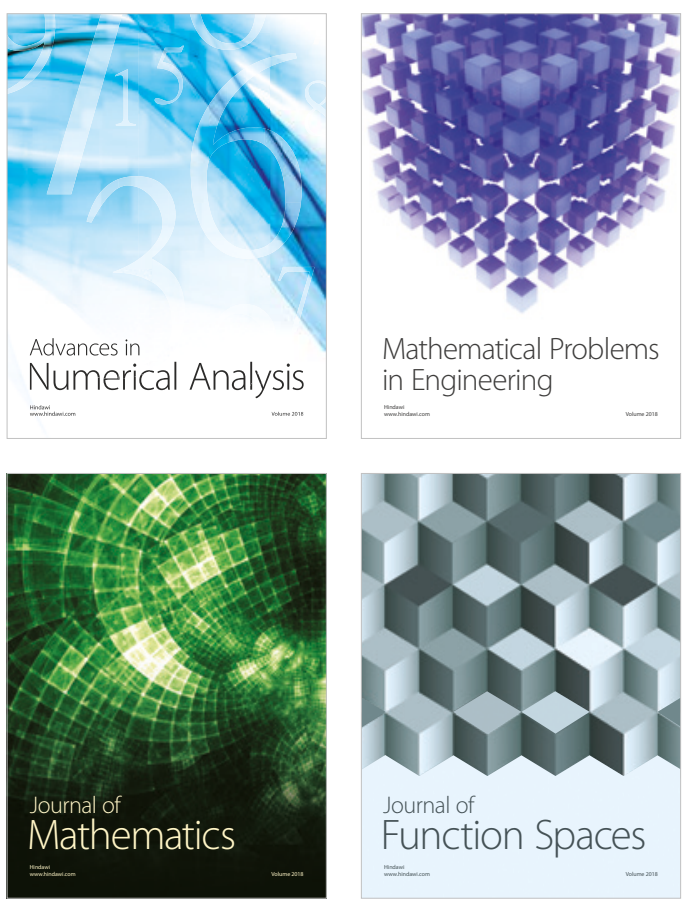

Mathematical Problems in Engineering

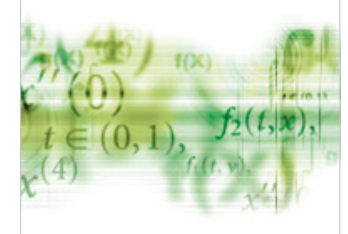

International Journal of

Differential Equations

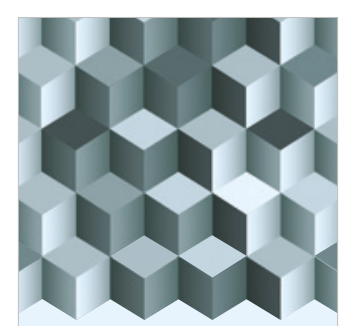

Journal of

Function Spaces
The Scientific

World Journal

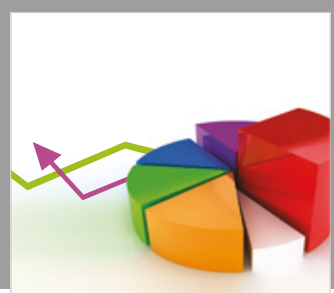

Journal of

Probability and Statistics
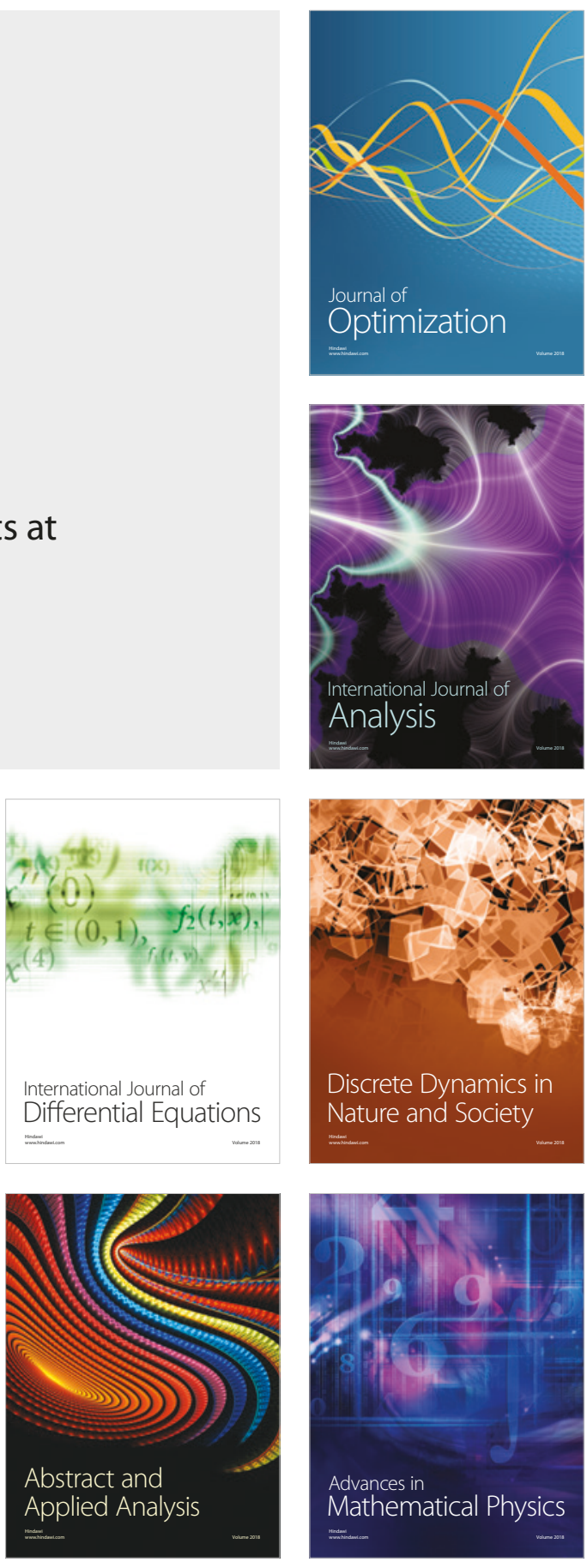